IJIDeM @ Springer-Verlag, 2008. This is a post-peer-review, pre-copyedit version of an article published in the International Journal on Interactive Design and Manufacturing, Vol. 2, No. 3, pp. 175-182. The final authenticated version is available online at: http://dx.doi.org/10.1007/s12008-008-0046-4

\title{
Tangible user interface integration in engineering
}

\author{
Nadine Couture · Jeremy Legardeur • \\ Guillaume Riviere
}

\begin{abstract}
In this paper we describe the design and the development of two specific tangible user interface (TUI) platforms. The aim of the first one is to support computer aided design (CAD) parts assembly operations in the mechanical product domain. The aim of the second one is mainly designed to help stakeholders during the task of validation of subsoil model in the field of geosciences. In this paper, we propose a design methodology of the tangible parts (also called props) based on our previous experiences in TUI development. This methodology is mainly based on multidisciplinary work and user tests and we assume that this process is reproducible.
\end{abstract}

Keywords Tangible user interface (TUI) - Experiences feedback · Multidisciplinary design methodology · CAD parts assembly $\cdot$ Geosciences

\section{Introduction}

New Computer Human Interaction systems have recently appear proposing new concepts for hardware as well as for software. Among these new technologies, the tangible user interface (called TUI in following) seeks to make intuitive interfaces in order to combine the physical world and the digital one to simplify interaction. TUI systems are based on the use of real and physical objects which allow a representation of the data and/or a physical control of digital information. In 1997, Ishii and Ullmer [7] gave a first vision of tangible inter-

\footnotetext{
N. Couture $(\varangle) \cdot$ J. Legardeur · G. Riviere

ESTIA, Technopôle Izarbel, 64210 Bidart, France

e-mail: n.couture@estia.fr

J. Legardeur

e-mail: j.legardeur@estia.fr

G. Riviere

e-mail: g.riviere@estia.fr
}

action: "To make computing truly ubiquitous and invisible, we seek to establish a new type of HCI that we call "Tangible User Interfaces". TUIs will augment the real physical world by coupling digital information to everyday physical objects and environments". In 2000 in [14] they characterized more particularly the tangible parts of the TUI, also called props, as a way to "give physical form to digital information, employing physical artifacts both as representations and controls for computational media". In 2002 Aliakseyeu et al. [1] and then Subramanian in 2004 [13] proposed props specific to navigation and manipulation of 3D data which are the general context of the TUI design presented in this article.

The research question we tackled is the design and development of TUI dedicated to industrial domains in order to perform one or more complex tasks.

Our standpoint is that in specific and industrial domains, any TUI has to be designed in collaboration with people who have the knowledge and know-how. On this point we can cite [3] "A real-world application domain has specific properties, constraints, and requirement that should be taken into account when designing interaction techniques for that domain". Thus, the developments of TUI systems adapted to specific industrial tasks are often complex as it implies different skills to design all the system components.

This paper focuses on the design phase of the development of TUI systems. Indeed, the development of a TUI system implies the design of different components:

- The tangible parts (called props in this paper) which are the physical components of the system. These props are the entities that will be manipulated by the users.

- The software and hardware systems that will be used to track the positions and movements of props.

- The interface system that provides to the user a feedback of the props operations in its environment. 
No design method is yet established for designing TUIs. Interested reader can consider a state of the art about this subject in $[5,6]$.

In this paper, the design of the software, hardware, and interface systems will not be here detailed as these points are very specific to each application. Indeed, there are lots of possibilities of software and hardware tracking systems (video capture, electronic connection, contactor devices, magnet systems...) as well as multi-sensorial feedback systems (computer screen, head-mounted device, sound and force feedback and vibration based system,...).

We focus on the design phase of the tangible parts of the TUI system. Indeed, in most of TUI based system, there are one or more tangible objects (props) that must be designed according to the specificity of the targeted task. Our objective is to propose a methodology to define the main characteristics of any props. This methodology is based on our previous TUI projects developed at ESTIA engineering institute: ESKUA and GeoTUI (see Fig. 1).

The design of props appropriated for the application domain is one of the major difficulties for the creation of the communication interface between human and data since there are huge possibilities concerning both form and functionalities. In 1999, Pierce et al. [10] recognized that the use of accessories is determining for the manipulation of digital data, and they showed that is not possible to provide a generic accessory that represents all virtual objects.

Assuming that the task is correctly defined, we propose a methodology based on two different user-centred iterations, in order to obtain the set of pertinent props.

1. Define the set of possible props (called $S_{\mathrm{pp}}$ in the following)

2. Refine $S_{\mathrm{pp}}$ to obtain the right set of props by testing evaluation validation. This refined set is the set of validated props (called $S_{\mathrm{vp}}$ in the following)

For the first iteration we present two approaches: a formal one and a second one based on a creative method.

For the second iteration, our standpoint is that it is necessary to lead user-evaluation. The aim of user evaluation is to validate the usability of props for the specified task (preliminary defined). Under the term "usability" we consider the definition due to Hix and Hatson and given in [3, p. 7]: "the characteristics of an artefact (here the props) that affect the user's use of the artefact [...] including ease of use, user task, user comfort, and system performance"

\section{Design process for $S_{\mathrm{pp}}$}

We propose two complementary approaches. The first one is based on creative methods. The second one is a formal one for a specific area: mechanical assembling.

\subsection{Pluridisciplinary approach}

The development of TUI system is complex as it implies different skills to design all the system. As we said before, one key point in the perspective of TUI system development is the design of props.

During our previous projects concerning TUI development, we mainly used a common methodology based on different steps to define the props form and functions:

\subsubsection{Observation of the targeted task with their existing practices}

The main objective of this step is to observe the users in their "normal" environment without the use of any TUI tool. This first works allows us to identify some problems and needs with the use of existing tools and methods. The main objective is to identify where the TUI could provide the most significant benefit for the users on different aspects: cognitive, increase collaboration, simplification of the use of existing systems... The idea is to identify and evaluate the gap proposed by the TUI regarding the existing tools and methods traditionally used during the studied task. After this analysis, the TUI proposal can be defined:

- As a new system that solves unrealized operations by existing tools or methods.

- As a new system to replace existing tools or methods.

- As a new system to complete existing tools on specific operations.

This first step allows defining the main needs of the TUI system.

\subsubsection{Benchmarking of existing approaches}

The main objective of this step is the identification of the existing tools or methods that could be used or adapted for the props forms and functions definition. The main objective is to identify existing concepts of methods that can be used or adapted to define the general form and functions of the props. This step can be achieved by the identification and the proposition of well-known methods (such as Functional analysis) of engineering design domain or specific tools and methods proposition (such as DFA Design for Assembly for ESKUA application, as we can see further).

\subsubsection{Brainstorming of TUI design}

Based on the results of the previous step, this stage aims to define the general specifications of the props according to the identified needs for the TUI. The objective is twofold. The first one concerns the definition of the main forms of the 
Fig. 1 ESKUA (a) and GeoTUI (b)
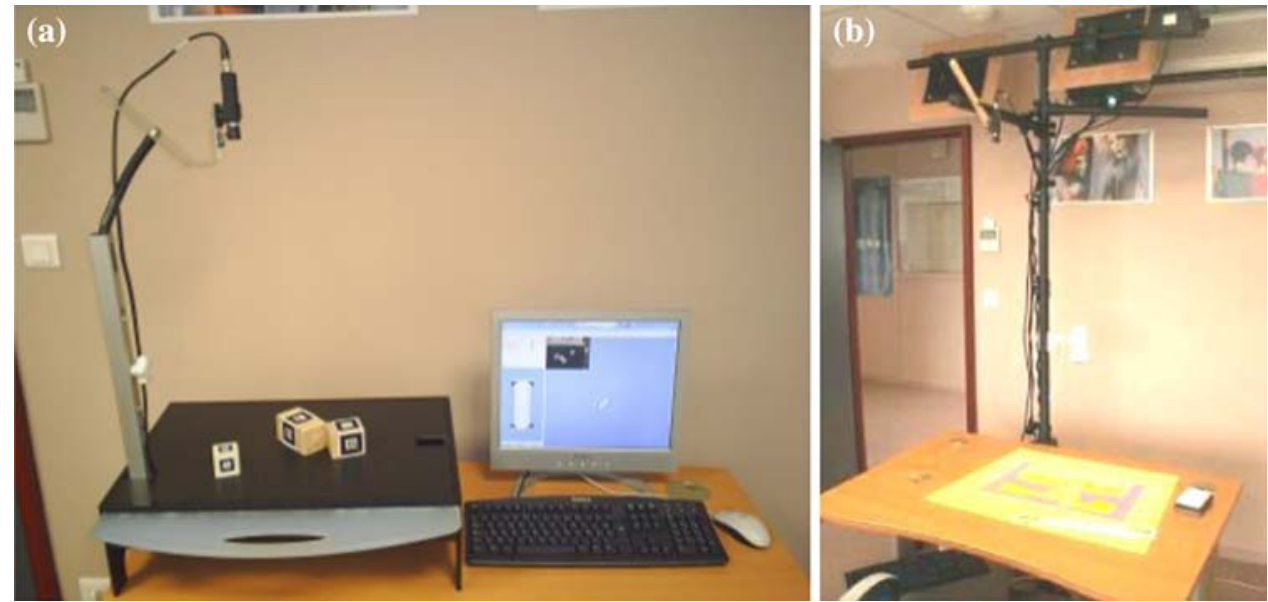

props. The second one concerns the definition of the main functions of the props. This point implies to define the different actions that could be done with the props.

\subsection{Formal approach}

The idea is to propose a reusable methodology based on filters in order to define relevant tangible parts for a TUI. We focus on the specific area of the mechanical assembly, but we believe that it is possible to reuse in other areas since the designers of TUI (include end users) are able to define the right criteria. The right criteria are those which lead the user in her/his task (for our case study, see Sect. 2 below).

To summarize, the methodology steps are the following (detailed in [4]):

1. Get a relevant basic component set of the domain. This set must contain the most used elements, in the assembly of the addressed area.

2. Categorize each element of the set, in regards to the exhibited criteria: number and type of functional surfaces, direction of the surfaces normal, direction of the revolution surface axis.

3. Gather together all the components that share the same value for the criteria. This step gives component families from which props will be extracted: their shape design is led by the criteria values. Thus, each prop is an abstraction of a whole set of components, in regards to their assembly properties.

At this step, the found out families might be too numerous again. Then, we recommend filtering the props with a loop on the set: "while the number of the props is greater than 20 , remove the props that are a combination of $n$ props" ( $n$ starting value is 2 , and is increased of one at each iteration). It seems that a set of 20 elements is a good compromise to keep the system usable, even if this value has not been experimentally proved.

\section{User evaluation to obtain $S_{\mathrm{vp}}$}

For the second iteration, our standpoint is that it is necessary to lead user evaluation. As we said before, the aim of user evaluation is to validate the usability of props for the specified task (preliminary defined). Evaluation from ergonomics criteria is strongly complementary to user evaluation. However, ergonomics criteria efficiency, well known in evaluation system, has still to be proved in design. As a matter of fact, the difficulty comes from the fact that in design the object to evaluate is uncompleted. Moreover we assume that each user evaluation could be (would be!) an inspiration source for the design of other props it will be necessary to build. We consider user evaluation as a supplementary way to put end user in the design process.

Then, in order to validate the right set of props, we propose user evaluations tacking into account summative evaluations and interviews.

\section{Application of design methodology on two industrial cases}

4.1 ESKUA, French acronym that means "experiment of a usable tangible system for assembling"

\subsubsection{The targeted problem and the task}

ESKUA is an operational platform based on TUI dedicated to interact with computer aided design (CAD) software. ESKUA, thanks to its props, provides to the designers a physical simulation of parts assembly operations. The use of this platform gives the designer a physical perception of the assembly constraints during the "virtual" CAD parts manipulations. The ESKUA platform is designed in order to capture the actions (displacement, assembly, rotation, etc.) that the user will carry out on the real objects. The capture of the 
position and the orientation of the props are based on a specific video capture system.

\subsubsection{ESKUA $S_{\mathrm{pp}}$ by DFA method, $S_{\mathrm{pp} 1}$}

We focused our research principally on design for assembly (DFA) methods [2]. The main goal of these DFA methods is to identify the assembly criteria and to enable their anticipation from the design step in order to engage precautionary actions as soon as possible. The DFA methods enable to determine an aptitude coefficient for the manual assembly of a product based on the evaluation of basic operations concerning the main operations (grasping, handling, insertion) on the parts. During DFA analysis, the user associates simple volumetric shapes to the CAD parts such as cubes, long or short cylinders, and parallelepipeds. This principle was our starting point for the constitution of a first set of props (see Fig. 1) that is detailed in a previous work [8].

\subsubsection{ESKUA $S_{\mathrm{pp}}$ by formal approach $S_{\mathrm{pp} 2}$}

This theoretical study of assembling task is based on Rejneri definitions [11]. It confirms that, in the context of an assembling process, an object can be fully represented by its functional surfaces: planes, cylinders, cones and spheres. Then, from contacts between two surfaces, all of the eleven common functional joints can be built, and any assembling task can be simulated. This preliminary work exhibits criteria that characterize an object in the context of assembling. Then, our approach proposes to categorize a relevant part set of the addressed area compared with these theoretical criteria. The so-built methodology ensures that the generated prop set is able to represent any assembling task, as far as the starting part set is representative of the mainly used parts of the domain. The first step removes each part surface that is never used for assembling; then, it enumerates each functional surface, taking into account the number and the gender; finally, it determines the normal vector of functional surfaces. As a result, the methodology leads to identify each part with assembling criterion. At each step, parts sharing the same value for the defined criterion are gathered together in new subsets. Finally, the props are built from this analysis, as abstractions of each subset properties.

In order to validate the proposed approach, the methodology has been used to find out a props set for mechanical parts assembling. It is based on the analysis of 600 parts from the ICS classification 21 and exhibits 12 props.

So as to test this methodology, we have applied it to the particular domain of mechanical part assembly. For this experimentation, we have chosen to use the ISO normalized components (21st section of the ICS: mechanical systems and components of general use). There are more than 600 and are relevant of the basic components used in mechanical assembly. Figure 2 shows that filtering the first set is required, as it contains 61 elements.

Figure 3 shows that filtering several times the initial set allows decreasing the number of elements.

We plan to build these props, to test them in a real context and to compare the result with the use of the first set $S_{\mathrm{pp} 1}$.

\subsubsection{ESKUA user evaluation to obtain $S_{\mathrm{vp}}$ by refining $S_{\mathrm{pp} 1}$}

In this section, we present the user evaluation of our props and the evolution on props that it provides.

Our principal goal of the user-study was to verify the following hypothesis:

- Does the utilization of the props induce a reflection on the assembly?

- Does the link between the prop and the CAD part depend on the shape or on the functional surfaces?

- Does the user naturally create new props when the parts have more complex shapes?

The classical method that we followed to get the experimental protocol consists of the following steps:

1. Elaboration of the assembly task.

2. Validation of the assembly task by the reference user.

3. Precise refinement of the assembly task.

4. For all subjects of the evaluation, do:

- First interview to characterize the test subject.

- The assembly task is presented to the test subject in the test environment in written and oral form until acknowledgement of understanding.

- Camera-recorded execution of the test with verbalization of the user subject.

- At the end of the test, second interview to get the impressions of the test subject.

5. Analysis of the recorded tests and interviews, synthesis of the results, and propositions.

The following six persons completed this test: one reference user, two CAD experts, one assembly expert, one accustomed CAD user, and one ergonomist. The principal assembly task is "the assembly with tangible props of a CAD model". For this, we provide a 3D visualization of a CAD assembly and the set of props. Then we ask the user to realize the assembly by using the props. This was done for ten different assembly tasks.

In these ten different assembly tasks, we can qualitatively distinguish that the subjects have two ways to associate the assembly of the props with the assembly of the CAD parts. The first association is based on reasoning on the geometric 
Fig. 2 The first props set (61 elements)

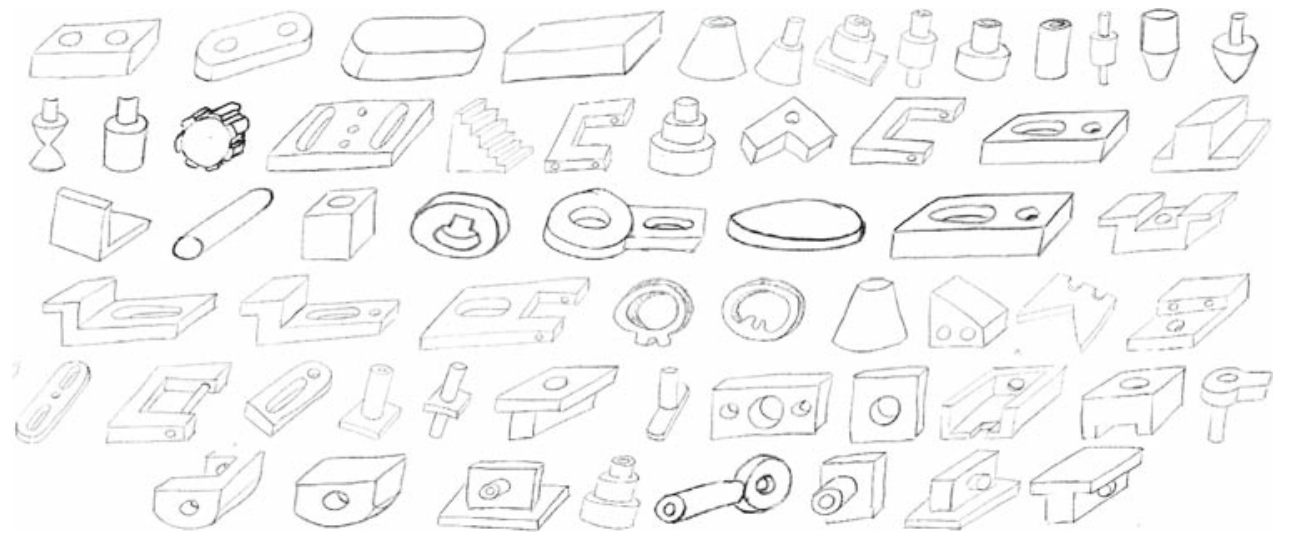

Fig. 3 The final props set (16 elements)
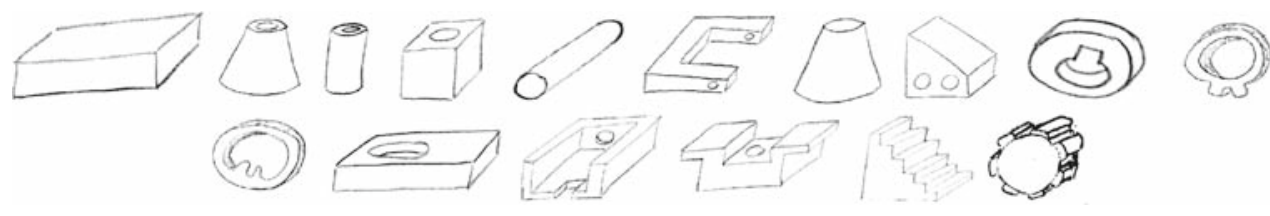

II I I t

shapes, and the second one is based on reasoning on the functional surfaces of the CAD parts.

In particular, it appears that the assembly expert (and this is the one we address with our research) tries to identify primarily the functional surfaces in order to analyse and optimize the product as a whole. The main result is that the less the users are specialized in the assembly task, the less they are reasoning about the surfaces and the more they reason about the geometries.

Our experiments highlight that subjects use different cognitive ways to associate CAD part with props. Basically, our experiments show that subjects propose different props combinations according to two kinds of criteria. The first criterion is the general form of the part (as DFA principle) and the second one is the functional surfaces of the part. In that sense, we decided to focus on the evolution of the TUI in order to foster associations between CAD parts and props with a functional surfaces based reasoning. Therefore, we have done a state of the art concerning the technology components mainly used in different mechanical products. These previous works allow us to propose a new set of props (see Fig. 4) with specific functional surfaces commonly used in assembly process such as: chamfer on shaft and bore (see no. 1, 4), fillet (see no. 7), flat on shaft (see no. 5, 4), key and keyway (see no. 4), groove and groove shoulder (see no. 5, 2), housing shoulder and recess (see no. 7), shaft shoulder (see no. 1), guide slot on a plane surface (see no. 9, 10,11,12), internal and external spline or multiple splines (see no. 2, 3), spot facing (see no. 7), mortice (see no. 8), spigot joint (see no. $6+8$ ).

Moreover, new "fastening props" had been also designed to allow the use of different fastening technology such as: bolt with nut, screw, centring pin, rivet, external and internal retaining ring, pin, positioning dowel, and hole-pin joint.

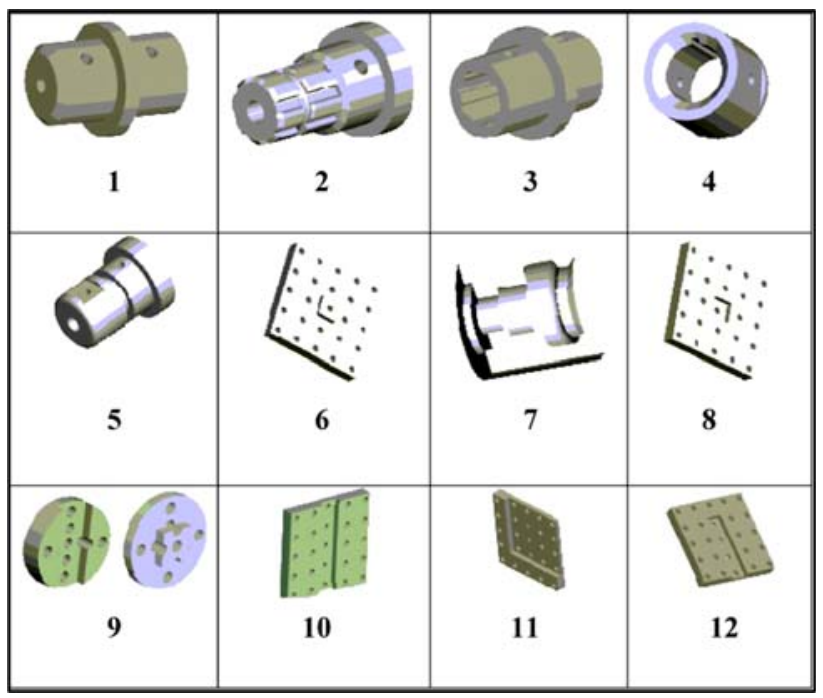

Fig. 4 ESKUA $S_{\mathrm{vp}}$

Based on this previous work, we developed additional props graft (see example on Fig. 5) in order to promote props combinations regarding both functional surfaces-based reasoning and geometric form reasoning.

\subsection{GeoTUI}

\subsubsection{The targeted problem and the task}

In this TUI, we address the problem of geosciences people, who encounter difficulties when using GUIs. People from IFP (French Oil Institute) explained us such problems. Difficulties are due to two principal facts: geoscientists manipulate huge and very complex data sets; and moreover, persons 


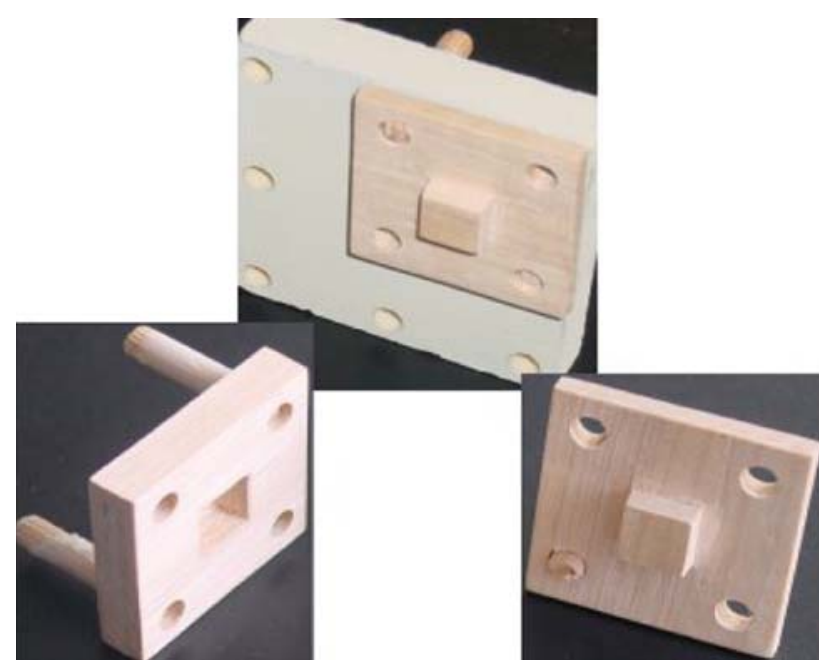

Fig. 5 Example of props with guide slot graft

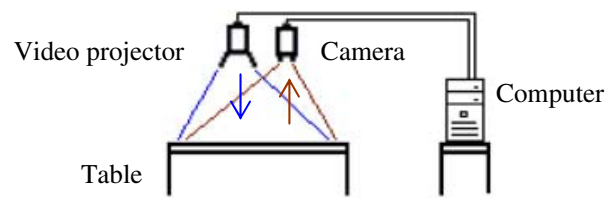

Fig. 6 A magic table system

from different specialities must often work in co-present collaboration. The work of geoscientists was already identified as an exploration field for TUIs in 2002, by Aliakseyeu et al. [1]. They mention the work on seismic recordings as an application field.

When iterating for reconstructing a subsoil model, geophysicists and geologists often fail to validate their hypothesis. Our partners from IFP desire to develop new interaction tools. They already tried a VR immersive room. But success is not there: too many constraints appeared. Worn devices, like HMD or data-gloves, make concentration or collaboration difficult. The expensive cost and the space occupied (one specific big room) do not allow owning more than one. Users must respect a booking scheme, and are not free to merge this tool with their daily work. The fact is that user gave up the room. Our proposition of TUI involves targeting support of co-present collaboration work, and simplification of the interaction. Nevertheless, this TUI must respect some constraints from IFP. It must be quite cheap and light, to spread it, and integrate it, in every office.

To work with a subsoil model, geoscientists and geologists use geographic maps and geologic plane cuts. The ordinary configuration to interact is composed of two big computer screens, a mouse and a keyboard. But even two screens are not enough to easily interact with huge subsoil plane cuts. And when three or four persons work in co-present collaboration, screens are too difficulty reachable to show a detail to another colleague. Likewise mouse and keyboard are difficultly shared.

The foundation of our TUI is a magic table (see on Figs. 1b, 6). Geographic maps and geological plane cuts are displayed through video projection at the surface of the tabletop. Users can interact at the surface of the table with objects. Perception space and action space coincide. Information is displayed where user looks at, so ergonomic criteria from [12] are respected. Tabletops give a quite large common display surface. They also give a large common space, where users can share props. These conditions of work bring closer with selfinduced conditions of work known by geoscientist before the arrival of computers in their office. But, of course, continuing to work with the benefits of the computer calculation.

The task we focused in is to select a cut line from a geographic map, in order to obtain a geological cut plane of the subsoil model studied. The aim of this task is more than simple navigation in the subsoil. This task is central to geoscientist's tasks, because it allows doing other trade tasks which are done from cut planes. So selecting a cut line is done very often, and it is important to make this task very easy to do.

\subsubsection{GeoTUI $S_{\mathrm{pp}}$ obtained by creative approach}

Following the creative approach presented above in section "design process for $S_{\mathrm{pp}}$ ", we first observe the users with the existing GUI. With the GUI, to choose a cut line from a geographic map, a user must successively select two points using the mouse. When the first point is put, the line can only rotate around this point when putting the second point. Then to adjust the position of the cut line, the user must select back the first point to move it, and so on.

Our first proposition of tangible prop is an ordinary $40 \mathrm{~cm}$ long flat ruler, called $r$, made in transparent plastic (see on Fig. 7a). The system is not precise enough, and for the moment we augmented displaying a digital line which shows the exact selected position. Intrinsically, the ruler controls directly every movements of the cut line. Thus, with one or two hands, the user can directly choose the cut line position.

Our second proposition of prop is a small puck, called $p$, with a diameter of $35 \mathrm{~mm}$ and a depth of $10 \mathrm{~mm}$, made in wood. As for the mouse, the user must control successively two points to move the cut line. These solutions are not retained because it does not change anything compared to the mouse.

Our third proposition of prop is two pucks, called pp, used both to control a digital line displayed through them. This prop is totally mixed. Moving one puck cannot enable the control of all possible movements of the cut line. Nevertheless it becomes possible with two hands. 
Fig. 7 a First proposition: manipulate a ruler to select cut line from displayed map. b A physical validation pad
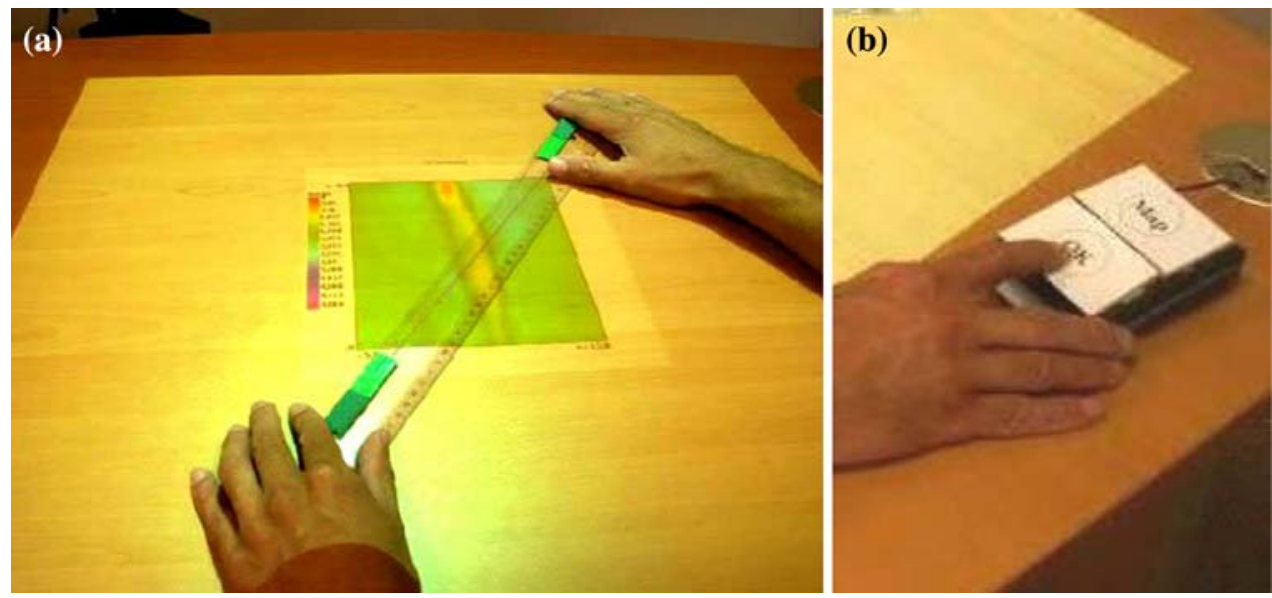

At this point of the design,

$S_{\mathrm{pp}}=\{r, p, \mathrm{pp}\}$

\subsubsection{GeoTUI user evaluation to obtain $S_{\mathrm{vp}}$ by refining $S_{\mathrm{pp}}$}

The first and recent user evaluations of the task "selecting cut lines" were made. Ten persons from IFP were the users. Two scenarios were made in order to compare our TUI with the IFP's GUI. Between tests on TUI and GUI, only the user interface differs: the same geological calculation software computes maps and cut planes, and the scenarios are identical. Each user realizes the two scenarios on the two interfaces. Note that using an interface before another can damage observation results. To balance this fact, GUI then TUI is the order for half of the users, and TUI then GUI is the order for the other half.

The first scenario is composed of five small exercises. The observant asks the tester to make six cut planes from the geological map at six precise positions. In the second scenario, the tester must find impact points of reflectors present in the model of subsoil. The tester is free to navigate in the model as he wants. When he finds one point, he must write down the name of the impact on a blank sheet of paper, also present on the table.

Concerning the TUI, we also want to observe how users understand and learn the TUI. Nowadays, not yet many people are informed about this kind of systems. A brief explanation of the functioning must be made: "The camera watches your actions. The video projector displays on the table the results of your actions, by example maps or plane cuts. To interact with the system you have props. They look like small objects. Here, they are in the box. Take them and install yourself to get ready for exercises." Yet no explanation is made about the way to interact. Thus the observant ask the user to do the first cut plane in $Y=3 \mathrm{~km}$ with no more explanations. The tester has three kinds of props in front of him: one ruler, two pucks, and a small validation pad (see on Fig. 7b) composed of two buttons "OK" and "Map".

Statistic analyses are work in progress. But let us give first observations. A $100 \%$ chose the ruler to interact. Then we conclude

$S_{\mathrm{vp} .}=\{r\}$

Nevertheless several users did not use immediately the validation pad. But finally every tester took it in hand very rapidly. We can already say that users, right handed or left handed, used either one hand or two hands to manipulate the ruler. They also use more often two hands (i.e. bimanual interactions with one prop) when they need precision and more often one hand when they travel in the model. We also observe automation from several users, using one hand for the validation pad, and the other for the ruler (i.e. bimanual interaction with two props of two different kinds). These first feedbacks from users are very encouraging. They conclude "it is towards this sort of interface we have to go"

This test was a mono-user one. The next one will be for a collaborative task. This task will be done by several users, who will have to work on the subsoil model from cut planes.

\section{Conclusion}

The number of props of a TUI must be limited in order to avoid a complex interaction with the users. We proposed in this paper a design methodology to define the form and the number of props of a TUI. The application of the method is illustrated by two TUIs that have been developed at ESTIA engineering institute.

Adding a specific prop for one task must be justified by an improvement of the interaction. For example, the ruler of GeoTUI seems to be an appropriated prop for the task of selecting a cut line from a map. On one side, all users 
associated this prop to this task. On another side, the ruler permits to directly control all movements of the cut line. Thus, even if pucks are more generic props, which can be used in other tasks, we validate the choice of the ruler for the task of selecting a cut line, which is central to the geoscientists work.

With the ESKUA platform, the designer will associate props to CAD parts, and handle these physical objects to carry out the assembly of the product. So the user is confronted with real assembly operation constraints such as parts positioning difficulties or maintaining element in a joint position. Our new props set based on functional surfaces reasoning allow carrying out physical simulations while enabling designers to identify assembly difficulties, and to modify parts design. We plan to follow the ideas of [9] in order to lead experimentations and to explore results on the benefits of TUIs on tabletops at a cooperative and collaborative point of view for our concrete application fields.

Acknowledgments We thank every person from ESTIA who took part to user evaluations on ESKUA. We also want to thank Jacques Jacobs and his colleagues from IFP for fruitful discussions, and every person from IFP who participated to user evaluations on GeoTUI. Special thanks to our colleagues Olivier Zephir who helped us to manage experimentations with end users and to Fabrice Depaulis to contribute to the formal approach.

\section{References}

1. Aliakseyeu, D., Subramanian, S., Martens, J.B., Rauterberg, M.: Interaction Techniques for Navigation through and Manipulation of 2D and 3D Data. In: Proc. EGVE'02, Eurographics Association, pp. 179-188 (2002)

2. Boothroyd, G., Dewhurst, P.: Product Design for Assembly-A Designer's Handbook. University of Massachusetts (1993)
3. Bowman, D.A., Kruijff, E., LaViola, J.J., Poupyrev, I.: 3D User Interfaces: Theory and Practice. Addison Wesley, Reading (2005). ISBN 0-201-75867-9

4. Depaulis, F., Couture, N., Legardeur, J., Garreau, L.: A reusable methodology based on filters in order to define relevant tangible parts for a TUI. In: Proc. Electronic Imaging 2005, Society of Photo Optical, vol. 5664, pp. 530-539 (2005)

5. Hornecker, E.: A Design Theme for Tangible Interaction: Embodied Facilitation. In: Proc. ECSCW’05, pp. 23-43. Springer, New York (2005)

6. Hornecker, E., Buur, J.: Getting a Grip on Tangible Interaction: A Framework on Physical Space and Social Interaction. In: Proc. CHI 2006, pp. 437-446. ACM Press, New York (2006)

7. Ishii, H., Ullmer, B.: Tangible Bits: Towards Seamless Interfaces between People, Bits and Atoms. In: Proc. CHI'97, pp. 234-241. ACM Press, New York (1997)

8. Legardeur, J., Garreau, L., Couture, N.: Experiments to evolve toward a tangible user interface for CAD parts assembly. In: Proc. Electronic Imaging 2004, vol. 5291, pp. 438-445. Society of Photo Optical (2004)

9. Maher, M.L., Kim, M.J.: Studying Designers Using a Tabletop System for 3D Design. In: Proc. Tabletop 2006, pp. 105-112. IEEE Computer Society (2006)

10. Pierce, J., Stearns, B., Pausch, R.: Two Handed Manipulation of Voodoo Dolls in Virtual Environments. In: Proc. Symposium on Interactive 3D Graphics 1999, pp. 141-145. ACM Press, New York (1999)

11. Rejneri, N.: Détermination et simulation des opérations d'assemblage lors de la conception de systèmes mécaniques. $\mathrm{PhD}$ thesis, Institut National Polytechnique de Grenoble, France (2000)

12. Scapin, D.L., Bastien, J.M.C.: Ergonomics criteria for evaluating the ergonomic quality of nteractive systems. Behav. Inf. Technol. 6, 220-231 (1997)

13. Subramanian, $\mathrm{S} .:$ Tangible Interfaces for Volume Navigation. $\mathrm{PhD}$ thesis, University of Technology Eindhoven, The Netherlands (2004)

14. Ullmer, B., Ishii, I.: Emerging frameworks for tangible user interfaces. IBM Syst. J. 39(3, 4), 915-931 (2000) 